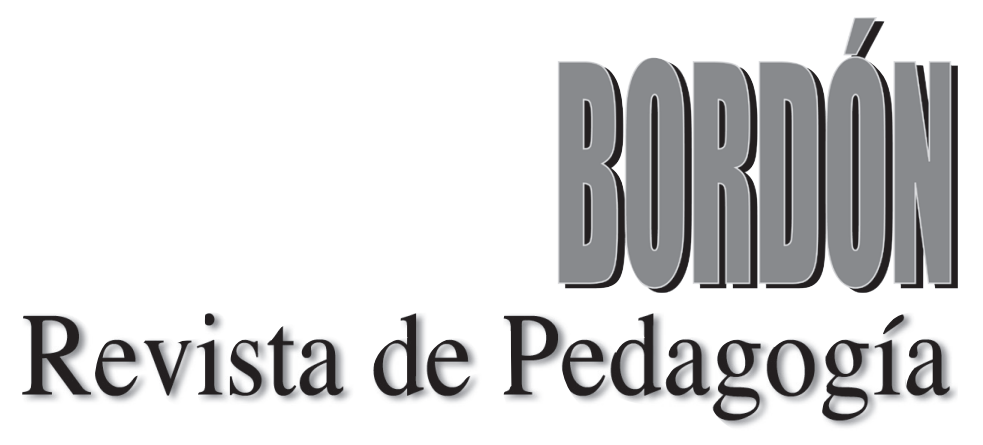

NÚMERO MONOGRÁFICO / SPECIAL ISSUE

TIC y educación inclusiva / ICT and inclusive education

Verónica Marín-Díaz

(editor invitado / guest editor)

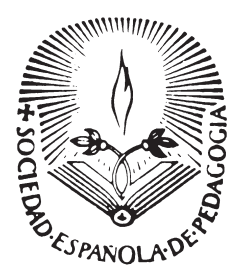

Volumen 69

Número, 3

2017 


\title{
CONOCIMIETTOS SOBRE EL TRASTORNO POR DÉFICIT DE ATENCIÓn CON HIPERACTIUIDAD (TDAH) En FUTUROS PROFESIONALES DEL ÁmBITO ESCOLAR
}

\section{Knowledge of Attention-Deficit/Hyperactivity Disorder [ADHD] among future school professionals}

\author{
JAVIER GIL FLORESY ALEJANDRO MEGÍAS IGLESIAS \\ Universidad de Sevilla
}

DOI: 10.13042/Bordon.2017.50278

Fecha de recepción: 24/05/2016 - Fecha de aceptación: 06/02/2017

Autor de contacto / Corresponding Author: Javier Gil Flores. E-mail: jflores@us.es

INTRODUCCIÓN. El Trastorno por Déficit de Atención con Hiperactividad (TDAH) presenta una importante prevalencia en la población escolar. El alumnado con este trastorno encuentra dificultades para el aprendizaje, que conducen a un menor rendimiento educativo. Por ello, los profesionales presentes en el contexto escolar han de poseer una formación adecuada para el diagnóstico y tratamiento del TDAH. La investigación sobre los conocimientos de los profesionales acerca del TDAH ha sido escasa en nuestro país y se ha centrado únicamente en el profesorado, bien en ejercicio o en formación. En este estudio se pretende valorar los conocimientos sobre TDAH que poseen los futuros profesionales, incluyendo psicólogos, pedagogos y maestros. Se analizan además las diferencias en función de la titulación y de la experiencia con individuos afectados por TDAH. MÉTODO. Se utilizó una muestra de 497 estudiantes que cursan el último año de las titulaciones de psicología, pedagogía y educación primaria en la Universidad de Sevilla. Los conocimientos sobre TDAH fueron medidos a partir de la Knowledge of Attention Deficit Disorders Scale. Para el análisis de datos se utilizaron estadísticos descriptivos (porcentajes, medias y desviaciones típicas) y pruebas no paramétricas para el contraste de grupos. El tamaño de los efectos se calculó mediante el estadístico r. RESULTADOS. Los profesionales en formación poseen buenos conocimientos sobre síntomas y diagnóstico del TDAH. En cambio, presentan carencias importantes respecto al tratamiento y, en particular, sobre características generales, causas y consecuencias. Los mayores niveles de conocimientos corresponden a los futuros psicólogos y a quienes poseen experiencia con individuos afectados por TDAH. DISCUSIÓN. A partir de estos resultados, se sugiere la necesidad de mejorar la formación inicial universitaria de los profesionales presentes en el ámbito escolar, para que puedan atender eficazmente a las necesidades del alumnado con TDAH en los centros escolares.

Palabras clave: Educación especial, Trastorno por Déficit de Atención con Hiperactividad, Nivel de conocimientos, Estudiantes de pregrado. 


\section{Introducción}

En la última edición del Diagnostic and Statistical Manual of Mental Disorders (American Psychiatric Association, 2013) se revisa la definición del Trastorno por Déficit de Atención con Hiperactividad (TDAH) caracterizándolo como un patrón de conducta, presente en múltiples contextos, que puede dar lugar a problemas de rendimiento en los ámbitos social, educativo o laboral. Se trata de un trastorno del neurodesarrollo que se inicia en la infancia y puede continuar para algunas personas durante la etapa adulta. Implica síntomas persistentes y generalizados de desatención, hiperactividad e impulsividad, que incluyen conductas tales como falta de atención sobre los detalles, dificultad para organizar tareas, hablar en exceso, inquietud o incapacidad para permanecer sentado cuando es necesario.

El TDAH afecta a un importante número de personas. Aunque disminuyen con la edad, los síntomas del TDAH persisten en la adolescencia para un $85 \%$ de los diagnosticados en la infancia y para un $65 \%$ en la edad adulta (Faraone, Biederman y Mick, 2006). Pero sin duda, el estudio del TDAH en niños y adolescentes ha concentrado la mayor parte de la investigación sobre este trastorno. Los estudios de prevalencia del TDAH en población escolar han venido indicando que entre el 3\% y $7 \%$ de los individuos estarían afectados. En estos márgenes se sitúan las más recientes estimaciones. Polanczyk, Willcutt, Salum, Kieling y Rohde (2014), tras una exhaustiva revisión, seleccionaron 154 estudios sobre TDAH en individuos con una edad máxima de 18 años, realizados en países de todo el mundo entre 1985 y 2012. Mediante técnicas meta-analíticas, cifran en $5,47 \%$ la prevalencia del TDAH a nivel mundial. Esta cifra viene a coincidir con la aportada por la American Psychiatric Association (2013), que sitúa en aproximadamente el 5\% los individuos en edad escolar afectados. La alta prevalencia hace que el TDAH constituya uno de los trastornos más comunes en la infancia y en la adolescencia.
El TDAH conlleva efectos negativos sobre las conductas y actividades desarrolladas en el ámbito familiar, escolar o social. Centrándonos en el contexto escolar, la desatención, impulsividad e hiperactividad, que constituyen síntomas centrales del TDAH, afectan especialmente al comportamiento y a la trayectoria académica de los niños, disminuyendo la autorregulación y las habilidades de aprendizaje (Barkley, 2013). El alumnado con TDAH encuentra dificultades para participar en actividades que exigen prestar atención, seguir instrucciones o permanecer sentado. Ello reduce su capacidad de aprendizaje, conduciendo a un menor rendimiento escolar y menos posibilidades de éxito académico (Martínez, Herrera y Peyres, 2014; Montague, Enders y Castro, 2005).

Teniendo en cuenta su alta prevalencia y las consecuencias que conlleva para los individuos afectados, el TDAH es uno de los trastornos infantiles más estudiados. La profusión de trabajos publicados sobre este tema refleja el interés que suscita en la comunidad científica. Frente a la investigación sobre causas, etiología, diagnóstico y tratamiento, son menos frecuentes los trabajos centrados en el estudio de los niveles de conocimiento sobre el TDAH. Parte de la investigación sobre este tópico ha abordado el estudio de los conocimientos, concepciones o actitudes de los propios niños o adolescentes y de sus padres, de los trabajadores sociales o de los profesionales de la salud (Bussing et al., 2012; Díaz, 2015; Moldavsky y Sayal, 2013; West, Taylor, Houghton y Hudima, 2005).

La literatura incluye también trabajos sobre el nivel de conocimientos de individuos con perfiles profesionales más directamente vinculados al contexto escolar, que constituyen el foco de atención en el presente trabajo. En el caso de nuestro sistema educativo, los profesionales que desde los centros escolares intervienen en el diagnóstico o tratamiento de alumnado con TDAH son fundamentalmente los maestros y orientadores, papel este último desempeñado por pedagogos o psicólogos. En 
diversos trabajos se ha examinado el nivel de conocimientos de profesionales de la psicología o pedagogía, siempre en comparación con otros agentes que intervienen dentro o fuera del ámbito escolar. Dryer, Kiernan y Tyson (2006) encontraron concordancias entre psicólogos y médicos en lo que respecta a las características y causas del TDAH, mientras que los maestros poseen sobre estos temas mayor número de creencias erróneas que los dos grupos anteriores. Weyandt, Fulton, Schepman, Verdi y Wilson (2009) afirmaban que, si bien los psicólogos escolares deberían estar provistos de información relevante y actualizada sobre el diagnóstico y tratamiento del TDAH, la investigación sobre conocimientos de los psicólogos era prácticamente inexistente. En consecuencia, llevaron a cabo uno de los escasos estudios realizados sobre este colectivo, confirmando la hipótesis de que los psicólogos escolares poseen un mayor nivel de conocimientos sobre TDAH que los maestros generales o especialistas en educación especial. Resultados similares encontraron Massé, Couture y Anciaux (2010), situando a los psicólogos por encima de los trabajadores sociales o profesionales de la salud, cuyos conocimientos sobre TDAH superan a su vez a los de maestros, maestros especialistas, directores escolares o pedagogos.

El nivel de conocimientos de los maestros es, sin duda, el tópico que más atención ha suscitado entre los investigadores. Los trabajos que abordan su estudio son mucho más numerosos que los dedicados a otros profesionales que intervienen desde el ámbito social, familiar, escolar o médico. Ello refleja el papel relevante de estos profesionales y la necesidad de que posean la formación suficiente para participar en la detección e intervención con el alumnado aquejado de TDAH. En general, los estudios realizados sobre maestros constatan un moderado o bajo nivel de conocimientos (Anderson, Watt, Noble y Shanley, 2012; Sciutto et al., 2016; Soroa, Gorostiaga y Balluerka, 2016; Vereb y DiPerna, 2004), coincidiendo en señalar mayores carencias en lo que respecta a etiología del TDAH que al conocimiento de los síntomas básicos y su tratamiento. En la misma dirección se han venido orientando los resultados obtenidos al explorar los conocimientos de los futuros maestros, quienes presentan también carencias relevantes (Akram, Thomson, Boyter y McLarty, 2009; Bekle, 2004; Canu y Mancil, 2012; Jarque y Tárraga, 2009; Liang y Gao, 2016).

En el reciente estudio transnacional llevado a cabo en nueve países por Sciutto et al. (2016), se constató que formación y experiencia previa con TDAH son buenos predictores del conocimiento de los maestros sobre este trastorno. Es decir, los conocimientos de los maestros mejoran tras haber tenido experiencia con alumnado afectado de TDAH (Kos, Richdale y Jackson, 2004) y cuando han recibido una formación adicional sobre el tema (Jones y Chronis-Tuscano, 2008). En nuestro país, Jarque, Tárraga y Miranda (2007) también han constatado mayores niveles de conocimiento entre los profesores que han tenido experiencia con alumnado afectado por TDAH.

Para diagnosticar el TDAH se requiere que sus síntomas se manifiesten en dos o más contextos (American Psychiatric Association, 2013), siendo el hogar y la escuela donde más fácilmente pueden constatarse. Por ello, el diagnóstico requiere que el personal médico recabe información de las familias y también de los profesionales presentes en el ámbito escolar, a quienes se debe la detección de una buena parte de los casos de TDAH. Hemos destacado ya el papel relevante del profesorado, que constituye la primera fuente de información sobre las conductas de los alumnos en el aula. Además, en nuestros centros educativos, interviene en el diagnóstico de alumnado con TDAH el personal que desempeña funciones de orientación, generalmente psicólogos o pedagogos.

El contexto escolar es un marco privilegiado, no solo para el diagnóstico de este alumnado, sino también para su tratamiento mediante una intervención adecuada desde las aulas (DuPaul 
y Stoner, 2003), que favorezca su progreso académico, social y personal. Aunque la mayor parte de la intervención sobre individuos con TDAH se ha dirigido al tratamiento de los síntomas conductuales (falta de atención, impulsividad o hiperactividad), es fundamental atender específicamente a los problemas asociados en el entorno escolar. Para ello, los profesionales que intervienen en este ámbito han de poseer conocimientos suficientes sobre el TDAH.

En el contexto español, la escasa investigación sobre conocimientos acerca del TDAH se ha centrado sobre los maestros en ejercicio (Jarque et al., 2007; Martínez et al., 2014; Soroa et al., 2016), o bien en la comparación de sus conocimientos con los que poseen maestros en formación (Jarque y Tárraga, 2009). Sin embargo, no hemos encontrado estudios sobre los conocimientos de otros profesionales presentes en nuestros centros escolares. En este trabajo, abordamos el análisis de los conocimientos sobre TDAH de los futuros profesionales, con el propósito de cubrir la ausencia de investigación al respecto y proveer una base empírica útil de cara a orientar su formación inicial. De acuerdo con ello, planteamos los siguientes objetivos:

a) Describir los conocimientos sobre TDAH en futuros profesionales del ámbito escolar.

b) Comparar el nivel de conocimientos sobre TDAH entre futuros profesionales de distintas titulaciones y entre futuros profesionales con o sin experiencia previa en este trastorno.

\section{Método}

Se ha adoptado un diseño descriptivo transversal, basado en el método de encuesta.

\section{Participantes}

Han participado en la investigación 497 estudiantes que cursaban el último año de las titulaciones de Grado en Educación Primaria (25,6\% de la muestra), Pedagogía $(42,7 \%)$ y Psicología (31,8\%) en la Universidad de Sevilla. La selección de estos se realizó mediante muestreo no probabilístico por conveniencia, invitando a participar al alumnado de diferentes grupos de clase en cada una de las titulaciones consideradas. El 83,1\% de los participantes son mujeres, frente a solo el 16,9\% de hombres, reflejando el tradicional predominio femenino en estas titulaciones. Sus edades se encuentran comprendidas entre los 20 y 53 años, con una media de 22,7 años (DT=4.0). El 15,5\% ha tenido experiencia en la intervención con afectados por TDAH.

\section{Instrumento}

Hemos utilizado la Knowledge of Attention Deficit Disorders Scale (KAADS), elaborada por Sciutto, Terjesen y Frank (2000). Se trata de un cuestionario diseñado para medir el nivel de conocimientos sobre el TDAH, que ha sido ampliamente empleado en trabajos anteriores con maestros en formación o en ejercicio. Además, fueron solicitados a los participantes datos demográficos (sexo y edad) y se les preguntó sobre su experiencia previa con individuos afectados por este trastorno. Esta variable se midió de manera dicotómica, indicando si los participantes habían tenido o no experiencia previa. Tratándose de futuros profesionales, su experiencia con afectados por TDAH se limita a la que hubieran podido adquirir durante la realización de prácticas externas, por lo que será probablemente escasa. No obstante, esta característica permite diferenciar entre dos subgrupos de participantes, cuyos conocimientos sobre TDAH podrían diferir.

La KAADS consta de un total de 36 ítems que recogen enunciados acerca del TDAH, ante los cuales los participantes han de responder si se trata de aseveraciones verdaderas o falsas, existiendo la opción de respuesta "no lo sé". Al considerar esta última opción, es posible diferenciar 
entre aquello que los individuos no saben (lagunas de información) y lo que representa un conocimiento incorrecto que lleva a considerar verdadera una afirmación que en realidad es falsa, o viceversa (concepciones erróneas). Los ítems de la KADDS se centran en tres áreas específicas: a) conocimientos generales sobre la naturaleza, causas y consecuencias del TDAH (15 ítems), b) síntomas/diagnóstico del TDAH (9 ítems) y c) tratamiento del TDAH (12 ítems). La estructura de la escala en estas tres áreas fue validada por sus autores (Sciutto et al., 2000) mediante el juicio de 40 expertos del ámbito de la psicología clínica y escolar. Cada experto ubicó los ítems en una de las tres áreas. La asignación de un ítem se dio por buena cuando al menos el $75 \%$ de los jueces coincidieron en su apreciación.

De cara a medir los conocimientos sobre TDAH, las respuestas son puntuadas con 1 en el caso de acierto y 0 cuando la respuesta es errónea o implica una laguna de conocimiento. La puntuación total en el cuestionario, obtenida como suma de las respuestas correctas, puede ir de 0 a 36 puntos. En la muestra utilizada, la consistencia interna de la KAADS se ha cifrado en un alfa de Cronbach de .80 , próximo al valor .86 hallado por Sciutto et al. (2000) con una muestra de maestros de educación primaria, o al valor .89 obtenido para la adaptación española del instrumento (Jarque et al., 2007).

\section{Procedimiento}

La aplicación de los cuestionarios se realizó mediante encuestadores que acudieron a las aulas universitarias para recoger las respuestas de los participantes. Previamente se había contactado con el profesorado implicado, solicitando el acceso a los grupos de clase. Los estudiantes fueron informados acerca de la naturaleza del estudio y el carácter voluntario de su participación en el mismo. Se garantizó el anonimato y se les solicitó sinceridad en las respuestas. Transcurrido un periodo no superior a diez minutos desde su distribución, el encuestador recogió los cuestionarios completados por los estudiantes.

\section{Análisis de datos}

El análisis descriptivo de los conocimientos sobre TDAH se ha basado en la distribución de frecuencias para las respuestas a los ítems, así como el cálculo de los estadísticos media y desviación típica para el número de aciertos en la escala. Debido a que es diferente el número de ítems en las áreas de conocimientos generales, síntomas/diagnóstico y tratamiento, las puntuaciones directas obtenidas como número de aciertos no son comparables. Por ello, hemos asignado a cada individuo una puntuación en cada subescala, obtenida como porcentaje de ítems de esa subescala respondidos correctamente. De este modo, las puntuaciones de un individuo en cualquiera de las subescalas están comprendidas entre 0 y 100 . La misma transformación ha sido realizada con el número total de aciertos en la escala.

De cara a la realización de comparaciones entre grupos, se aplicó inicialmente la prueba de Kolmogorov-Smirnov. Los resultados de esta prueba no permitieron mantener el supuesto de normalidad de las puntuaciones $(\mathrm{p}<.01)$. En consecuencia, se optó por pruebas no paramétricas. Las diferencias entre las tres áreas de conocimientos, planteada en el primer objetivo del estudio, se contrastaron mediante el análisis de la varianza para más de dos muestras relacionadas de Friedman, seguido de comparaciones post-hoc con la prueba de Wilcoxon.

En respuesta al segundo de los objetivos, se calcularon estadísticos descriptivos (media y desviación típica) para las puntuaciones en las tres áreas en función de la titulación y la experiencia previa en TDAH. Las diferencias en función de la titulación se valoraron mediante el análisis de la varianza por rangos de Kruskal-Wallis, seguido de comparaciones post-hoc con la U de MannWhitney para valorar las diferencias entre cada 
pareja de titulaciones. La prueba de Mann-Whitney también fue empleada para analizar las diferencias en función de la experiencia previa. El tamaño del efecto asociado a estas comparaciones se calculó mediante el índice r.

Todos los análisis se realizaron mediante el programa SPSS 23.

\section{Resultados}

La media para el número de ítems acertados por los futuros profesionales al responder a la escala KAADS ascendió a 17,9 (DT=5,6), lo cual supone el conocimiento de aproximadamente la mitad de los 36 ítems de que consta el instrumento. No obstante, este resultado puede ser matizado al calcular las puntuaciones, en una escala de 0 a 100 , para cada una de las tres áreas específicas consideradas. La media de estas puntuaciones se sitúa en los valores 74,3 (DT=19,5) para síntomas/diagnóstico, 45,8 (DT=19,8) para tratamiento, y solo 38,3 (DT=17,7) para conocimientos generales. Los resultados de aplicar a estos valores la prueba de Friedman indican diferencias significativas entre las puntuaciones logradas por los estudiantes en los tres ámbitos específicos a los que hacen referencia las subescalas $\left(\chi^{2}=556.9 ; \mathrm{p}<.001\right)$. Las comparaciones post-hoc mediante la prueba de Wilcoxon mostraron diferencias significativas $(\mathrm{p}<.001)$ en todos los casos, con moderados o altos tamaños de los efectos, comprendidos entre $\mathrm{r}=.42$ (diferencia entre conocimientos generales y tratamiento) y $\mathrm{r}=.85$ (diferencia entre conocimientos generales y síntomas/diagnóstico).

Para los tres tipos de estudiantes por separado, el perfil de conocimientos es similar al observado en la muestra global. En educación primaria, la media más alta se registra en síntomas/diagnóstico $(77,6)$ y la más baja en conocimientos generales $(35,7)$. El mismo patrón se registra entre los estudiantes de pedagogía (medias 65,2 y 30,8 en síntomas/diagnóstico y conocimientos generales, respectivamente) y entre estudiantes de psicología (medias 83,8 y 50,1 ).

Centrándonos en las respuestas obtenidas para cada ítem (tabla 1), constatamos que los niveles de conocimiento más elevados corresponden a los síntomas y diagnóstico del TDAH. En esta área, el porcentaje de aciertos supera el 77\% para 6 de los 9 ítems propuestos. En particular, la distracción frecuente (ítem 3) y el carácter inquieto (ítem 9) son los síntomas más conocidos, identificados por más del 90\% de los participantes. En cambio, las mayores tasas de concepciones erróneas o lagunas de conocimiento se registran en el ítem referido a la edad en que han de manifestarse los síntomas del TDAH para poder diagnosticar este trastorno (31,3\% de error y $29,8 \%$ de respuestas no lo sé en el ítem 5).

Tabla 1. Porcentajes de aciertos, errores y respuestas no lo sé

Ítem *

Acierto Error No lo sé

Conocimientos generales

1. La mayoría de las estimaciones sugieren que el TDAH se produce en aproximadamente el 15\% de los niños en edad escolar (F)

$16,8 \quad 42,7 \quad 40,5$

4. Los niños con TDAH suelen ser más obedientes con sus padres que con sus madres $(\mathrm{V})$

$4,4 \quad 63,2 \quad 32,3$

6. El TDAH es más común en los familiares biológicos de primer grado (p.ej., madre, padre) de los niños con TDAH que en la población general (V)

\begin{tabular}{|c|c|c|c|}
\hline 13. Un adulto puede ser diagnosticado TDAH (V) & 59,8 & 17,5 & 22,7 \\
\hline $\begin{array}{l}\text { 17. Los síntomas depresivos son más frecuentes en niñas TDAH que en niños } \\
\text { NO-TDAH (V) }\end{array}$ & 24,8 & 30,2 & 45,0 \\
\hline
\end{tabular}


Tabla 1. Porcentajes de aciertos, errores y respuestas no lo sé (cont.)

Ítem *

Acierto Error No lo sé

19. La mayoría de los niños con TDAH "superan" sus síntomas al inicio de la pubertad y funcionan "de manera normal" en la edad adulta (F)

$26,6 \quad 37,7 \quad 35,7$

22. Si un niño con TDAH es capaz de demostrar una atención sostenida a los videojuegos o la televisión durante más de una hora, ese niño también es de mantener la atención durante al menos una hora de clase o tarea (F)

24. Un diagnóstico de TDAH por sí mismo hace a un niño susceptible de ser insertado en un aula de educación especial (F)

(26,6

27. Los niños con TDAH suelen experimentar más problemas en los contextos novedosos que en los contextos familiares $(F)$

$\begin{array}{lll}58,3 & 17,8 \quad 23,9\end{array}$

. Hay características físicas específicas que pueden ser identificadas por los médicos (por ejemplo, pediatra) y ayudan a hacer un diagnóstico definitivo de TDAH (F)

29. En edades escolares, la prevalencia del TDAH en niños y niñas es equivalente (F) $\quad 46,1 \quad 13,1 \quad 40,8$

30. En niños muy pequeños (menos de 4 años), los problemas de conducta en niños con TDAH (por ejemplo, hiperactividad, falta de atención) son claramente diferentes de las conductas apropiadas para la edad de los niños sin TDAH (F)

31. Los niños con TDAH se distinguen más fácilmente de los niños NO-TDAH en un aula que en el recreo del colegio (V)

32. La mayoría de los niños con TDAH evidencia cierto grado de bajo rendimiento escolar en los años de la escuela primaria (V)

$65,5 \quad 15,6 \quad 19,0$

$9,5 \quad 64,7 \quad 25,8$

$40,4 \quad 26,2 \quad 33,5$

$46,1 \quad 13,1 \quad 40,8$

$35,8 \quad 29,6 \quad 34,6$

33. Los síntomas del TDAH se ven a menudo en los niños sin TDAH que provienen de ambientes familiares inadecuados y caóticos (V)

Síntomas / diagnóstico

3. Los niños con TDAH se distraen frecuentemente con estímulos externos (V)

5. Para ser diagnosticado con TDAH, los síntomas del niño deben haber estado presentes antes de los 7 años (V)

7. Uno de los síntomas de los niños con TDAH es que han sido físicamente crueles con otras personas $(\mathrm{F})$

$62,1 \quad 20,3 \quad 17,6$

$69,0 \quad 12,8 \quad 18,2$

$29,7 \quad 35,6 \quad 34,7$

$29,7,7$

. Los niños con TDAH a menudo se mueven y retuercen en sus asientos (V)

11. Es común que los niños con TDAH tengan un sentido exagerado de autoestima o grandiosidad (F)

14. Los niños con TDAH a menudo tienen antecedentes de robo o de causar desperfectos físicos en las pertenencias de otras personas $(\mathrm{F})$

16. Los conocimientos actuales sobre el TDAH sugieren dos grupos de síntomas: falta de atención e hiperactividad/impulsividad (V)

21. Para ser diagnosticado como TDAH, un niño debe exhibir síntomas relevantes en dos o más ambientes (por ejemplo, casa y escuela) (V)

\begin{tabular}{ccc}
91,9 & 4,4 & 3,6 \\
\hline 38,9 & 31,3 & 29,8 \\
\hline 77,9 & 4,7 & 17,4 \\
\hline 91,5 & 5,0 & 3,4 \\
\hline 52,6 & 13,7 & 33,7 \\
\hline 60,8 & 16,9 & 22,3 \\
\hline
\end{tabular}

$85,9 \quad 3,6 \quad 10,5$

$80,6 \quad 6,0 \quad 13,3$

26. Los niños con TDAH presentan a menudo dificultades para organizar las tareas $\mathrm{y}$ actividades (V)

88,1

5,3

6,7

Tratamiento

2. La investigación actual sugiere que el TDAH es en gran parte el resultado de habilidades de crianza ineficaces $(F)$

$62,3 \quad 11,6 \quad 26,2$


Tabla 1. Porcentajes de aciertos, errores y respuestas no lo sé (cont.)

Ítem *

Acierto Error No lo sé

8. Los fármacos antidepresivos han sido eficaces para reducir los síntomas de muchos niños con TDAH (V)

$27,0 \quad 44,4 \quad 28,6$

10. La capacitación correcta de los padres y el maestro en el manejo de un niño con TDAH es generalmente eficaz cuando se combina con el tratamiento con medicamentos (V)

12. Cuando se termina el tratamiento de un niño con TDAH, es raro que los síntomas del niño vuelvan a reproducirse $(F)$

$66,6 \quad 4,5 \quad 28,9$

15. Los efectos secundarios de los medicamentos estimulantes utilizados para el tratamiento del TDAH pueden incluir insomnio leve y la reducción del apetito (V)

18. La psicoterapia individual, por lo general, es suficiente para el tratamiento de la mayoría de los niños con TDAH (F)

20. En los casos graves de TDAH, la medicación se utiliza a menudo antes de intentar otras técnicas de modificación de conducta (V)

23. Reducir la ingesta de azúcar en la dieta o los aditivos en las comidas suele resultar efectivo para reducir los síntomas del TDAH (F)

25. Los estimulantes son el tipo más común de medicamento utilizado para tratar a los niños con TDAH (V)

34. Las intervenciones psicológicas para los niños con TDAH se centran principalmente en los problemas del niño relacionados con falta de atención (F)

35. La terapia electro convulsiva (es decir, tratamiento de choque) se considera un tratamiento eficaz para los casos graves de TDAH (F)

$60,4 \quad 6,2 \quad 33,4$

$48,4 \quad 17,7 \quad 33,9$

$48,7=26,2=0.25,2$

$48,7-26,2 \quad 25,2$

$32,7 \quad 22,0 \quad 45,4$

$20,0 \quad 51,7 \quad 28,3$

6. Los tratamientos para este trastorno que se centran principalmente en el castigo se consideran los más eficaces en la reducción de los síntomas de TDAH (F)

* Entre paréntesis se indica si el enunciado es verdadero (V) o falso (F).

En los ítems referidos a conocimientos generales se alcanzan los porcentajes más bajos de acierto. Solo 4 de los 15 Ítems han sido respondidos correctamente por más de la mitad de los participantes (ítems 13, 22, 24 y 31, cuyos enunciados, respuestas correctas y porcentajes de acierto se incluyen en la tabla 1). Además, casi dos de cada tres estudiantes han errado los ítems 4 y 27. En el primero de ellos, los encuestados han considerado falso que los niños con TDAH se muestren más obedientes con sus padres que con sus madres. En el segundo de estos ítems, se refleja la falsa creencia de que los niños con TDAH presentan más problemas en situaciones nuevas que en situaciones familiares. La respuesta a 8 de los 15 ítems ha sido eludida por más de un tercio de los participantes (ítems 1, 6, $17,19,28,29,30$ y 33 , recogidos en la tabla 1 ).

Finalmente, los conocimientos sobre el tratamiento del TDAH se sitúan en niveles medios, con tasas de acierto superiores al 50\% en 5 de los 12 ítems correspondientes a esta área (ítems 2, $10,12,15$ y 36, mostrados en la tabla 1). Los principales errores detectados indican un desconocimiento del uso de estimulantes para tratar el TDAH (ítem 25) y la falsa creencia de que la intervención psicológica para estos niños se centra principalmente sobre los problemas de falta de 
TABLA 2. Estadísticos descriptivos para los conocimientos sobre el TDAH en función de titulación y experiencia previa

\begin{tabular}{cccc}
$\begin{array}{c}\text { Conocimientos } \\
\text { generales }\end{array}$ & $\begin{array}{c}\text { Síntomas / } \\
\text { diagnóstico }\end{array}$ & Tratamiento & Total \\
\hline Media (DT) & Media (DT) & Media (DT) & Media (DT) \\
\hline
\end{tabular}

Titulación

\begin{tabular}{lllllllll}
\hline Ed. Primaria & 35,7 & $(15,5)$ & 77,6 & $(15,8)$ & 43,2 & $(18,1)$ & 48,9 & $(13,3)$ \\
\hline Pedagogía & 30,8 & $(15,7)$ & 65,2 & $(21,4)$ & 37,4 & $(17,5)$ & 41,3 & $(14,1)$ \\
\hline Psicología & 50,1 & $(15,6)$ & 83,8 & $(13,1)$ & 58,9 & $(17,0)$ & 61,3 & $(11,1)$ \\
\hline Experiencia previa & & & & & & & & \\
\hline Sin experiencia & 37,6 & $(18,1)$ & 73,3 & $(19,8)$ & 44,9 & $(20,5)$ & 49,0 & $(15,9)$ \\
\hline Con experiencia & 44,0 & $(15,4)$ & 78,9 & $(14,5)$ & 50,7 & $(16,3)$ & 54,7 & $(12,5)$
\end{tabular}

atención (ítem 34). En ambos casos, los errores afectan a más de la mitad de los participantes.

La tabla 2 recoge los niveles de conocimiento en los subgrupos determinados por la titulación y la experiencia de intervención con individuos afectados por TDAH. Para los participantes en el presente estudio, las mayores puntuaciones en el total de la escala KAADS y en cada una de sus áreas corresponden a los futuros psicólogos y las más bajas a los futuros pedagogos. Las puntuaciones de quienes han tenido alguna experiencia con individuos afectados por TDAH superan a las obtenidas por quienes carecen de ella.
El análisis de los conocimientos acerca del TDAH en función de la titulación, llevado a cabo mediante la prueba de Kruskal-Wallis, arrojó diferencias significativas $(\mathrm{p}<.001)$ entre las tres titulaciones, en lo que respecta a conocimientos generales $\left(\chi^{2}=102.40\right)$, síntomas/ diagnóstico $\left(\chi^{2}=84.49\right)$, tratamiento $\left(\chi^{2}=108.05\right)$ y puntuaciones totales en la escala $\left(\chi^{2}=144.69\right)$. Los resultados de las comparaciones a posteriori basadas en la prueba U de Mann-Whitney se muestran en la tabla 3. Las mayores diferencias se dan entre las titulaciones de psicología y pedagogía, con tamaños de los efectos que oscilan entre los valores .46 y .63 .

Tabla 3. Prueba de Mann-Whitney (U) y tamaño del efecto (r) para las diferencias de conocimientos sobre TDAH entre titulaciones

\begin{tabular}{lccccc}
$\begin{array}{l}\text { Grupos } \\
\text { comparados }\end{array}$ & & $\begin{array}{c}\text { Conocimientos } \\
\text { generales }\end{array}$ & $\begin{array}{c}\text { Síntomas / } \\
\text { diagnóstico }\end{array}$ & Tratamiento & Total \\
\hline Ed. primaria / pedagogía & $\mathrm{U}$ & $9810.0^{*}$ & $8295.5^{* *}$ & $10178.0^{*}$ & $7494.5^{* *}$ \\
\hline & $\mathrm{r}$ & .16 & .30 & .16 & .27 \\
\hline Ed. primaria / psicología & $\mathrm{U}$ & $5060.5^{* *}$ & $7560^{*}$ & $5063.0^{* *}$ & $4181.5^{* *}$ \\
\hline & $\mathrm{r}$ & .40 & .19 & .41 & .45 \\
\hline Pedagogía / psicología & $\mathrm{U}$ & $5935.0^{* *}$ & $7515.5^{* *}$ & $6079.5^{* *}$ & $3824.0^{* *}$ \\
\cline { 2 - 6 } & $\mathrm{r}$ & .52 & .46 & .53 & .63
\end{tabular}

${ }^{*} p<.01 ; * * p<.001$ 
En el caso de la comparación entre quienes cuentan o no con experiencia previa, los resultados de la prueba de Mann-Whitney muestran diferencias significativas para las subescalas conocimientos generales ( $U=8651.5 ; \mathrm{p}=.004$; $\mathrm{r}=.14$ ) y tratamiento ( $\mathrm{U}=9435.0 ; \mathrm{p}=.024 ; \mathrm{r}=.11$ ), así como para las puntuaciones totales ( $\mathrm{U}=8113.5 ; \mathrm{p}=.009 ; \mathrm{r}=.13)$. No obstante, el tamaño del efecto medido por el estadístico $r$ es en los tres casos bajo.

\section{Discusión}

En el presente trabajo hemos analizado los conocimientos sobre el TDAH que poseen futuros profesionales del ámbito escolar. Los resultados indican un moderado nivel de conocimientos, pero con diferencias importantes entre las áreas consideradas. El nivel en síntomas y diagnóstico es claramente superior al obtenido en conocimientos generales y tratamiento, donde se sitúan las principales concepciones erróneas o lagunas de conocimiento. Este patrón de resultados se alinea con el obtenido en la primera aplicación de la KAADS por sus autores (Sciutto et al., 2000), y son consistentes también con los presentados por Jarque et al. (2007) para la versión española del instrumento. Teniendo en cuenta que nuestro estudio se centra en futuros profesionales, la principal implicación de estos resultados se enmarca en el contexto de su formación inicial. El contenido de la misma debería incluir con la suficiente amplitud y profundidad los conocimientos relativos al TDAH, focalizando especialmente la atención en los conocimientos generales sobre la naturaleza, causas y efectos del TDAH, y también en el tratamiento del alumnado que presenta este trastorno. Los resultados mostrados en el presente estudio han permitido identificar concepciones erróneas y lagunas de conocimientos que habrían de ser subsanadas en estudiantes de psicología, pedagogía y educación.

El análisis de los conocimientos sobre TDAH en función de titulación y experiencia ha revelado diferencias significativas en ambos casos. En lo que respecta a la titulación, los futuros psicólogos presentan un mayor nivel de conocimientos que los futuros pedagogos y maestros. Ello haría presagiar, de cara a su desempeño profesional, una mayor cualificación de estos profesionales para identificar alumnado con TDAH, o establecer y evaluar intervenciones eficaces. Nuestros resultados con profesionales en formación concuerdan con los obtenidos al valorar los conocimientos de profesionales en ejercicio. Así, por ejemplo, Dryer et al. (2006) encontraron mayor nivel de conocimientos en psicólogos y médicos que en maestros. Weyandt et al. (2009) también hallaron superioridad en los conocimientos de los psicólogos escolares frente al profesorado general y especialista en educación especial. En el estudio de Massé et al. (2009), los niveles más altos de conocimientos correspondieron a los psicólogos educativos y los más bajos al personal escolar compuesto por maestros, directores o pedagogos.

Aunque sensiblemente menores que en el caso de la variable titulación, la experiencia con individuos afectados por TDAH genera diferencias significativas en los conocimientos. La literatura ha venido señalando el papel de la experiencia como fuente de conocimiento sobre TDAH. Así, el nivel de conocimientos del profesorado es mayor cuando ha trabajado con alumnado afectado (Sciutto et al., 2000, Kos et al., 2004; Jarque et al., 2007; Jones y Chronis-Tuscano, 2008), o bien conoce algún amigo o familiar diagnosticado con TDAH (Sciutto et al., 2016). Los resultados del presente estudio hacen extensivo ese papel a los futuros profesionales del ámbito escolar, aunque matizando que en nuestro caso la experiencia previa es muy reducida y el efecto de esta variable es bajo.

A partir de las conclusiones presentadas en este trabajo, se deriva la necesidad de mejorar la formación de los futuros profesionales del ámbito escolar. Las instituciones de educación superior habrían de incorporar de manera 
efectiva contenidos formativos que incrementen los conocimientos sobre TDAH y las posibilidades de superar los problemas de aprendizaje que afectan a los individuos con TDAH en los centros escolares. Para ello, es necesario el desarrollo de competencias no solo para la detección y diagnóstico, sino también para la implementación de intervenciones en las aulas adaptadas a las necesidades de este tipo de alumnado.

Autores como Barkley (2004) o Weyandt et al. (2009) han señalado estrategias de intervención en las que profesionales tales como maestros, pedagogos o psicólogos habrían de estar formados. Algunas de estas estrategias, validadas empíricamente en la literatura, incluyen el uso de tareas prácticas que requieren respuestas activas, retroalimentación frecuente, refuerzo positivo ante las tareas realizadas, instrucciones explícitas, tareas académicas altamente estructuradas, materiales estimulantes (por ejemplo, añadiendo colores a los textos), división de las tareas amplias en unidades pequeñas de trabajo o enfoque multisensorial (por ejemplo, usando instrucciones escritas y verbales). Aspectos como la comunicación con las familias y el establecimiento de estrategias conjuntas de intervención son también áreas de formación relevantes para la capacitación de los futuros profesionales.

Sin embargo, para mejorar la formación de los futuros profesionales no basta la incorporación de este tipo de conocimientos y estrategias como contenidos formativos. Es necesario adoptar un enfoque de enseñanza-aprendizaje con mayor presencia de metodologías activas, que favorezca un adecuado desarrollo de competencias. En este sentido, la experiencia práctica habría de ganar terreno en la formación de los estudiantes. Nuestros resultados señalan que la experiencia con individuos afectados ejerce un efecto positivo sobre el conocimiento acerca del TDAH. Las prácticas externas que estudiantes de psicología, pedagogía o de los títulos de maestro realizan en centros escolares habrían de ser aprovechadas para favorecer el contacto con este tipo de alumnado, contribuyendo a una adecuada formación teórico-práctica. Ampliar la presencia de estas prácticas a lo largo del currículum seguido en la educación superior, sin limitarlas a los últimos cursos, podría contribuir a generar profesionales más competentes para el trabajo con alumnado afectado de TDAH.

Una buena formación de los profesionales sobre los síntomas y el tratamiento del alumnado afectado de TDAH potenciaría la intervención educativa, contrarrestando el elevado peso que la medicación tiene actualmente como estrategia para combatir este trastorno. En particular, es fundamental mejorar la formación de los pedagogos y maestros, quienes han evidenciado aquí las mayores carencias en cuanto a conocimientos sobre el TDAH. Más aún si consideramos que, en su futuro desempeño profesional, los maestros habrán de ser frecuentemente los primeros en sugerir la necesidad de diagnosticar a alumnos con TDAH (Sax y Kautz, 2003), además de implementar las intervenciones educativas en el aula y proporcionar retroalimentación sobre los progresos alcanzados en el tratamiento de este trastorno.

Entre las limitaciones del presente estudio se encuentra la utilización de un instrumento de medida para el cual sus autores, más allá del juicio de expertos, no aportaron información relevante sobre la validez del mismo. Además, la variable experiencia previa con sujetos TDAH fue medida a nivel dicotómico, sin permitir precisar el tipo de experiencia o el grado en que se posee. Otra limitación es la utilización de una muestra no aleatoria de alumnado circunscrita a la Universidad de Sevilla, lo que limitaría las posibilidades de generalizar los resultados a los futuros profesionales que se forman en el conjunto de las universidades españolas. La posibilidad de que la formación impartida en diferentes instituciones de educación superior pueda diferir en sus contenidos aconsejaría realizar estudios similares en otros contextos o con 
muestras más amplias, incluyendo alumnado de diferentes universidades. Líneas futuras de investigación para profundizar en los conocimientos sobre TDAH que poseen los diferentes actores implicados podrían centrarse también en el colectivo de padres y madres de este alumnado. La investigación sobre los conocimientos de los padres es muy escasa (West et al., 2005), a pesar del importante papel que pueden asumir en el diagnóstico y tratamiento del trastorno. Determinar el nivel de conocimientos en la diversidad de agentes implicados en la atención al alumnado afectado de TDAH es un primer paso para procurar la mejora en las áreas que resulten deficitarias. Un adecuado nivel de conocimientos contribuirá a optimizar las actuaciones educativas desarrolladas en los ámbitos escolar y familiar.

\section{Referencias bibliográficas}

Akram, G., Thomson, A. H., Boyter, A. C., y McLarty, M. (2009). ADHD and the role of medication: Knowledge and perceptions of qualified and student teachers. European Journal of Special Needs Education, 24, 423-436. doi:10.1080/08856250903223088

American Psychiatric Association (2013). Diagnostic and statistical manual of mental disorders (5th ed.). Washington, DC: Author.

Anderson, D. L., Watt, S. E., Noble, W., y Shanley, D. C. (2012). Knowledge of attention deficit hyperactivity disorder (ADHD) and attitudes toward teaching children with ADHD: The role of teaching experience. Psychology in the Schools, 49, 511-525. doi:10.1002/pits.21617

Barkley, R. A. (2004). Adolescents with attention-deficit/hyperactivity disorder: An overview of empirically based treatments. Journal of Psychiatric Practice, 10(1), 39-56.

Barkley, R. A. (2013). Taking charge of ADHD: The complete, authoritative guide for parents. New York, N.Y.: Guilford Press.

Bekle, B. (2004). Knowledge and attitudes about attention-deficit/hyperactivity disorder (ADHD): a comparison between practicing teachers and undergraduate education students. Journal of Attention Disorders, 7(3), 151-161. doi:10.1177/ 108705470400700303

Bussing, R., Zima, B. T., Mason, D. M., Meyer, J. M., White, K., y Garvan, C. W. (2012). ADHD knowledge, perceptions, and information sources: perspectives from a community sample of adolescents and their parents. Journal of Adolescent Health, 51 (6), 593-600.

Canu, W. H., y Mancil, E. B. (2012). An examination of teacher trainees' knowledge of attentiondeficit/hyperactivity disorder. School Mental Health, 4, 105-114. doi:10.1007/s12310-012-9071-3

Diaz, M. (2015). Facilitating urban school social worker collaboration with teachers in addressing ADHD: A mixed-methods assessment of urban school social worker knowledge. School Social Work Journal, 39(2).

Dryer, R., Kiernan, M. J., Tyson, G. A. (2006). Implicit theories of the characteristics and causes of attention-deficit/hyperactivity disorder held by parents and professionals in the psychological, educational, medical and allied health fields. Australian Journal of Psychology, 58(2), 79-82. doi:10.1080/ 00049530600730443

DuPaul, G. J., y Stoner, G. (2003). ADHD in the schools: Assessment and instructional strategies (2nd ed.). New York, NY: Guilford Press.

Faraone, S. V., Biederman, J., y Mick, E. (2006). The age-dependent decline of attention deficit hyperactivity disorder: A meta-analysis of follow-up studies. Psychological Medicine, 36(2), 159-165.

Jarque, S., y Tárraga, R. (2009). Comparación de los conocimientos sobre el Trastorno por Déficit de Atención con Hiperactividad (TDAH) de los maestros en activo y los futuros educadores. Infancia y aprendizaje, 32(4), 517-529. doi:10.1174/021037009789610421 
Jarque, S., Tárraga, R., y Miranda, A. (2007). Conocimientos, concepciones erróneas y lagunas de los maestros sobre el trastorno por déficit de atención con hiperactividad. Psicothema, 19(4), 585-590.

Jones, H. A., y Chronis-Tuscano, A. (2008). Efficacy of teacher in-service training for attentiondeficit/hyperactivity disorder. Psychology in the Schools, 45, 918-929. doi:10.1002/pits.20342

Kos, J. M., Richdale, A. L., y Jackson, M. S. (2004). Knowledge about attention-deficit/ hyperactivity disorder: a comparison of in-service and preservice teachers. Psychology in the Schools, 41(5), 517-526.

Liang, L., y Gao, X. (2016). Pre-service and in-service secondary school teachers' knowledge about attention-deficit/hyperactivity disorder (ADHD) and Attitudes toward students with ADHD. International Journal of Disability, Development and Education, 63(3), 1-15. doi:10.1080/103491 2X.2015.1123231

Martínez, M. T., Herrera, E., y Peyres, C. (2014). Los conocimientos del profesorado sobre TDAH y su relación con el rendimiento del alumnado. En J. Maquilón y N. Orcajada (eds.). Investigación e innovación en formación del profesorado (pp. 369-380). Murcia: EDITUM.

Massé, L., Couture, C., y Anciaux, V. (2010). Connaissances et croyances des intervenants des milieux scolaires, de la santé et des services sociaux à l'égard du trouble déficitaire de l'attention/ hyperactivité. Santé Mentale au Québec, 35(1), 153-179. doi:10.7202/044802ar

Moldavsky, M., y Sayal, K. (2013). Knowledge and Attitudes about Attention-deficit/hyperactivity disorder (ADHD) and its treatment: The views of children, adolescents, parents, teachers and healthcare professionals. Current Psychiatry Reports, 15(8), 1-7. doi:10.1007/s11920-0130377-0

Montague, M., Enders, C. K., y Castro, M. (2005). Academic and behavioral outcomes for students at risk for emotional and behavioral disorder. Behavioral Disorders, 31, 87-96.

Polanczyk, G. V., Willcutt, E. G., Salum, G. A., Kieling, C., y Rohde, L. A. (2014). ADHD prevalence estimates across three decades: an updated systematic review and meta-regression analysis. International Journal of Epidemiology, 43(2), 434-442. doi:10.1093/ije/dyt261

Sax, L., y Kautz, K. J. (2003). Who first suggests the diagnosis of attention-deficit/hyperactivity disorder? Annals of Family Medicine, 1, 171-174. doi:http://dx.doi.org/10.1370/afm.3

Sciutto, M. J., Terjesen, M. D., y Frank, A. S. (2000). Teachers' knowledge and misperceptions of Attention-Deficit/Hyperactivity Disorder. Psychology in the Schools, 37, 115-122. doi:10.1002/ (SICI)1520-6807(200003)

Sciutto, M. J., Terjesen, M. D., Kučerová, A., Michalová, Z., Schmiedeler, S., Antonopoulou, K., y Rossouw, J. (2016). Cross-national comparisons of teachers' knowledge and misconceptions of ADHD. International Perspectives in Psychology: Research, Practice, Consultation, 5(1), 34-50. doi:10.1037/ipp0000045

Soroa, M., Gorostiaga, A., y Balluerka, N. (2016). Conocimiento de los maestros sobre el TDAH: Relevancia de la formación y de las percepciones individuales. Revista de Psicodidáctica, 21(2). doi:10.1387/RevPsicodidact.14023

Vereb, R. L., y DiPerna, J. C. (2004). Teachers' knowledge of ADHD, treatments for ADHD, and treatment acceptability: An initial investigation. School Psychology Review, 33, 421-428.

West, J., Taylor, M., Houghton, S., y Hudyma, S. (2005). A comparison of teachers' and parents' knowledge and beliefs about attention-deficit/hyperactivity disorder (ADHD). School Psychology International, 26(2), 192-208. doi:10.1177/0143034305052913

Weyandt, L. L., Fulton, K. M., Schepman, S. B., Verdi, G. R., y Wilson, K. G. (2009). Assessment of teacher and school psychologist knowledge of Attention-Deficit/Hyperactivity Disorder. Psychology in the Schools, 46(10), 951-961. doi:10.1002/pits.20436 


\section{Abstract}

Knowledge of Attention-Deficit/Hyperactivity Disorder (ADHD) among future school professionals

INTRODUCTION. Attention Deficit Hyperactivity Disorder (ADHD) shows a significant prevalence in school population. Pupils with this disorder find difficulties to learn, and these drive to a lower educational achievement. Because of this, professionals who are present in the school context must have a proper education for diagnosing and treating ADHD. Research about professionals' knowledge on ADHD has been scarce in our country. It has only focused on teachers, not only on in-service teachers but also on preservice teachers. This work intends to describe the future professionals' knowledge about ADHD, including psychologists, pedagogues and teachers. It also aims to analyse the knowledge differences depending on degrees and experience in treating ADHD pupils. METHOD. We use a sample of 497 undergraduate students, on their last year of psychology, pedagogy and primary education degree from Seville University. ADHD knowledge is measured by the Knowledge of Attention Deficit Disorders Scale. The data analysis is based on descriptive statistics (percent, mean and standard deviation) and non-parametric tests for to compare groups. The effect size was calculated by $\mathrm{r}$ statistic. RESULTS. Future professionals show high knowledge of ADHD symptoms and diagnostic. However, they show important deficiencies in treatment and, remarkably, in general characteristics, causes and consequences. Future psychologists and those who have some experience in treating ADHD pupils show the highest levels of knowledge of this disorder. DISCUSSION. These results suggest a necessity to improve the initial training offered by higher education institutions for professionals acting in school context, in order to attend effectively the needs of ADHD students in schools.

Keywords: Special education, Attention Deficit Hyperactivity Disorder, Undergraduate students, Knowledge level.

\section{Résumé}

Connaissances des futurs professionnels du milieu scolaire sur le trouble de déficit de l'attention avec hyperactivité (TDAH)

INTRODUCTION. Le trouble de déficit de l'attention avec hyperactivité (TDAH) a une forte prévalence dans la population scolaire. Les élèves touchés par ce trouble ont des difficultés à apprendre, ce qui conduit à baisser les performances éducatives. En conséquence, les professionnels du milieu scolaire doivent posséder une formation adéquate pour le diagnostic et le traitement du TDAH. La recherche sur les connaissances des professionnels au sujet du TDAH se fait rare dans notre pays et elle a mis l'accent uniquement sur les enseignants, qu'ils soient en exercice ou en formation initiale. Cette étude vise à évaluer les connaissances sur le TDAH des futurs professionnels, y compris les psychologues, les pédagogues et les enseignants. En plus, les différences selon le diplôme et l'expérience avec les personnes touchées par le TDAH sont analysées. MÉTHODE. Un échantillon de 497 élèves de la dernière année des études universitaires de psychologie, pédagogie et enseignement primaire à l'Université de Séville a été utilisé. Les connaissances sur le TDAH ont été mesurées par la Knowledge of Attention Deficit Disorders Scale. Pour l'analyse des données, nous avons utilisé des statistiques descriptives (pourcentages, moyennes et écarts-types) et des tests non paramétriques pour la comparaison des groupes. Les 
effets observés ont été calculés en utilisant la statistique r. RÉSULTATS. Les futurs professionnels ont une bonne connaissance sur les symptômes et le diagnostic du TDAH. Cependant, ils ont des lacunes importantes dans le traitement et en particulier dans les caractéristiques générales, les causes et les conséquences. Les plus hauts niveaux de connaissances correspondent aux futurs psychologues et à ceux qui ont de l'expérience avec les personnes touchées par le TDAH. DISCUSSION. Au regard de ces résultats, il est suggéré d'améliorer la formation universitaire initiale des professionnels présents dans les écoles, afin qu'ils puissent répondre efficacement aux besoins des élèves atteints de TDAH.

Mots-clés: Éducation spécial, Trouble de Déficit de l'Attention avec Hyperactivité, Niveau de connaissances, Étudiants de premier cycle.

\section{Perfil profesional de los autores}

\section{Javier Gil Flores (autor de contacto)}

Catedrático de Universidad del Área de Métodos de Investigación y Diagnóstico en Educación, en la Universidad de Sevilla. Una parte relevante de su reciente producción científica se centra en el estudio de los procesos de enseñanza-aprendizaje y en el análisis de las características del profesorado. Correo electrónico de contacto: jflores@us.es

Dirección para la correspondencia: Facultad de Ciencias de la Educación. C/ Pirotecnia, s/n. 41003-Sevilla.

\section{Alejandro Megías Iglesias}

Graduado en Pedagogía por la Universidad de Sevilla. Ha sido alumno interno y becario de colaboración en el Departamento de Métodos de Investigación y Diagnóstico en Educación, de la Universidad de Sevilla.

Correo electrónico de contacto: alemegiasiglesias@gmail.com 